\title{
Regulation of vascular permeability in rat testes by modified human chorionic gonadotrophin
}

\author{
M. Veijola and H. Rajaniemi \\ Department of Anatomy, University of Oulu, Kajaanintie 52 A, SF-90220 Oulu, Finland
}

\begin{abstract}
Summary. High-performance liquid (HPL) -anion-exchange chromatography of testicular interstitial fluid (IF) and medium conditioned by polymorphonuclear leucocytes (PMN) revealed two major peaks (at fractions 2-3 and 7-8), which, with human chorionic gonadotrophin (hCG) increased vasopermeability in rat testes measured by the uptake of iodinated hCG and by interstitial fluid volume. When hCG was incubated with the fraction 7-8 peak and subsequently purified on HPLC it significantly increased testicular vasopermeability with a concomitant accumulation of PMNs in the testicular blood vessels and interstitium. The removal of hCG from the purified preparation with anti-hCG Sepharose 4B abolished the vasopermeability effect of the preparation, confirming that hCG itself is modified in such a way as to produce the response.

The results suggest that both IF and PMN-conditioned medium contain two components with different charges, which interact with hCG to increase vasopermeability by a PMN-mediated process. The results also indicated that hCG may itself be modified chemotactically, or so that it elicits production of leucoattractant in the testes.
\end{abstract}

Keymords: rat: testis; interstitial fluid; polymorphonuclear leucocytes; vasopermeability: leucoattractants

\section{Introduction}

Testicular interstitial fluid (IF) mediates endocrine interactions between the testicular cells and circulation, and paracrine interactions between the testicular cells. Thus, the factors controlling IF volume are of fundamental importance for testicular function. Luteinizing hormone (LH) and human chorionic gonadotrophin (hCG) increase testicular blood flow in rats (Damber et al., 1981; Setchell \& Sharpe, 1981; Widmark et al., 1986) and vascular permeability (Sharpe, 1979, 1981; Veijola et al., 1984) leading to accumulation of IF in the testes. It has been suggested that the increase in the permeability of the vascular wall endothelium is due to extravasation of polymorphonuclear leucocytes (PMNs) (Bergh et al., 1986) by a process in which, for example, plasminogen activator is involved (Veijola \& Rajaniemi, 1986; Loukusa et al., 1990). According to this concept, LH and hCG cause, as well as the steroidogenic response, formation of a leucotactic factor(s) in the testes. Because LH and hCG are known to regulate directly only Leydig cell function in the testes, they are the most likely source of such factors (Setchell \& Rommerts, 1985; Damber et al., 1987). We have shown that interaction of hCG with rat testicular IF produces, either directly or via some testicular cell, a leucotactic factor(s) (Veijola \& Rajaniemi, 1986). The mechanisms of this interaction are unknown, but it is likely that the component(s) in IF that, with hCG, gives rise to the leucotactic factor(s) originates in PMNs (Veijola \& Rajaniemi, 1989).

The present study elucidated by high-performance liquid (HPL)-anion-exchange chromatography, the component in interstitial fluid and PMN-conditioned medium interacting with hCG to 
produce leucotactic factor(s). The aim of the study was also to characterize further the process resulting in the formation of the leucotactic factor(s).

\title{
Materials and Methods
}

\begin{abstract}
Amimals and hormones. Adult male or female Wistar rats (75-90 days old), anaesthetized with ether, were used in the experiments. Highly purified hCG $\left(10900 \mathrm{iu} / \mathrm{mg}\right.$, Diosynth, Oss, Netherlands) was radioiodinated with $\mathrm{Na}^{125} \mathrm{I}$ (Amersham International plc, Bucks, UK) by the chloramine-T method (Greenwood et al., 1963) as described by Markkanen et al. (1980). The specific activity determined by self-displacement (Ketelslegers et al., 1975) ranged between 15 and $20 \mu \mathrm{Ci} / \mu \mathrm{g}$. The fraction of labelled hCG capable of binding to an excess of $\mathrm{LH} / \mathrm{hCG}$ receptor-rich ovarian membrane particles averaged $50-55 \%$. Nonspecific binding in the presence of a 1000 -fold excess of unlabelled hormone was $<1 \%$.
\end{abstract}

Isolation of PMN-leucocytes and production of the PMN-conditioned medium. Adult female rats were injected intraperitoneally with dehydrated Brewer thioglycollate medium (Difco Lab., Detroit, MI, USA). The peritoneal cavities were opened $24 \mathrm{~h}$ later, exudate was collected and the cells were purified by the Lymphoprep gradient (Nyegaard/Co., Oslo, Norway) as described by Veijola \& Rajaniemi (1989). Purified cells were $\sim 99 \%$ neutrophils, the rest of the cells being monocytes. The cells $\left(7 \times 10^{7} \mathrm{cells} / \mathrm{ml}\right.$ in phosphate-buffered saline (PBS), pH 7.4) were incubated for $2 \mathrm{~h}$ with slight agitation in a water bath at $35^{\circ} \mathrm{C}$ and pelleted thereafter by centrifugation at $300 \mathrm{~g}$ for $10 \mathrm{~min}$. Viability of the cells after incubation was $95 \%$, as determined by the Trypan blue, dye-exclusion test. The supernatants were further centrifuged at $106000 \mathrm{~g}$ for $2 \mathrm{~h}$ and then frozen at $-80^{\circ} \mathrm{C}$ until used in experiments.

Collection of testicular interstitial fluid. Testicular IF was collected from male rats as described by Pande et al. (1966). The testes were removed and a small incision was made carefully in the caudal end of the testicular capsule. They were then placed into $13 \times 100 \mathrm{~mm}$ conical polystyrene tubes and IF was allowed to collect at the bottom of the tubes over the next $16 \mathrm{~h}$ at $4^{\circ} \mathrm{C}$. The fluids were pooled and centrifuged for $2 \mathrm{~h} \mathrm{at} 106000 \mathrm{~g}$ and the supernatants frozen at $-80^{\circ} \mathrm{C}$ for further use.

Treatments of the testes. Testicular treatment was essentially the same in all experiments: $150 \mu \mathrm{l}$ of the medium to be tested was injected into the right testes; the left testes received the same volumes of control medium. Ninety minutes after the testicular treatments the animals were injected intravenously with ${ }^{125} \mathrm{I}-\mathrm{hCG}\left(2.0 \times 10^{6} \mathrm{c}\right.$.p.m. $)$ and killed $3 \mathrm{~h}$ later. The testes were removed and their radioactivity was measured in a $\gamma$-spectrometer (LKB-Wallac 1280 Ultrogamma, Wallac, Turku) and IF was collected and measured. Both ${ }^{125}$ I-hCG uptake and IF volume were calculated per unit weight of the testis.

High-performance liquid chromatography (HPLC) of IF and PMN-conditioned medium. The testicular IF and the PMN-conditioned media were subjected to HPLC at room temperature (Pharmacia LKB Biotechnology, Espoo, Finland) using a Bakerbond weak anion-exchange column (Wide-Pore PEI, $5 \mu \mathrm{m}, 4.6 \mathrm{~mm} \times 25 \mathrm{~cm}$ ) (J. T. Baker Chemicals B.V., Deventer, Netherlands). The column was equilibrated with $25 \mathrm{mmol}$ Tris- $\mathrm{HCl}$ buffer/l (pH 7.4). Samples ranged between 1.5 and $6.0 \mathrm{ml}$ and were loaded as doses of $1.5 \mathrm{ml}$ at intervals of $30 \mathrm{~min}$. After loading the samples, the column was eluted with a step gradient of $\mathrm{NaCl}$ from 0 to $2 \mathrm{~mol} / 1$ (as indicated in Fig. 1a) in $25 \mathrm{mmol}$ Tris/ $\mathrm{HCl}$ buffer $/ \mathrm{l}(\mathrm{pH} 7.4)$ in $70 \mathrm{~min}$. The flow was $0.5 \mathrm{ml} / \mathrm{min}$ and the elution was continuously monitored at $206 \mathrm{~nm}$. Ten fractions $\left(3.5 \mathrm{ml}\right.$ each) were collected, dialysed against water at $4^{\circ} \mathrm{C}$ for $24 \mathrm{~h}$ and lyophilized. Each fraction was dissolved in $300 \mu \mathrm{l}$ of PBS and tested as volumes of $150 \mu \mathrm{l}$ in the presence or absence of $5 \mathrm{ng}$ of hCG for permeabilityincreasing activity.

Fractions 7-8 of PMN-conditioned medium showing the highest permeability-increasing activity (see Fig. 1b) were pooled, dialysed overnight at $4^{\circ} \mathrm{C}$ against PBS ( $\mathrm{pH} 7 \cdot 4$ ), rechromatographed on HPLC and tested again in the presence or absence of $5 \mathrm{ng}$ of hCG for permeability-increasing activity (Fig. 2).

To elucidate whether hCG is converted chemotactically or whether the chemoattractant is formed from some components present in the PMN-conditioned medium, fractions 7 and 8 , which showed the highest permeability activity in the presence of hCG (see Fig. 1b), were pooled, dialysed overnight at $4^{\circ} \mathrm{C}$ against PBS (pH 7.4) and divided thereafter between two tubes. One tube was incubated for $2 \mathrm{~h}$ at $35^{\circ} \mathrm{C}$ with hCG $(100 \mathrm{ng}$ of unlabelled hCG +20000 c.p.m. of ${ }^{125} \mathrm{I}-\mathrm{hCG}$ ) and the other one, serving as a control, was incubated without hormone. After incubation, both samples were subjected to HPLC as described above. The fractions collected were dialysed against water at $4^{\circ} \mathrm{C}$ for $24 \mathrm{~h}$ and lyophilized and radioactivity was measured to locate the hCG-containing fractions. The lyophilized fractions were dissolved in PBS to give $5 \mathrm{ng} / 150 \mu$ in the peak fraction of hCG (fraction 2, see Fig. 3) and tested for permeability-increasing activity. The HPLC fractions of the control incubation (incubation without hCG) were processed simultaneously and were also tested for permeability-increasing activity in the presence of $5 \mathrm{ng}$ of negative hCG.

To elucidate further whether hCG itself is converted during incubation in such a way as to give rise to the permeability-increasing activity, fraction 2 (see Fig. 3) containing the hCG peak was treated either with anti-hCG Sepharose or control $\gamma$-globulin Sepharose 4B-conjugate. Anti-hCG $\gamma$-globulin and control $\gamma$-globulin Sepharose 4B-conjugates were prepared as described by Metsikkö \& Rajaniemi (1980). Half of fraction $2(300 \mu \mathrm{l})$ was incubated overnight at $4^{\circ} \mathrm{C}$ with $100 \mu \mathrm{l}$ of anti-hCG $\gamma$-globulin Sepharose 4B and the other half with the control Sepharose. The 


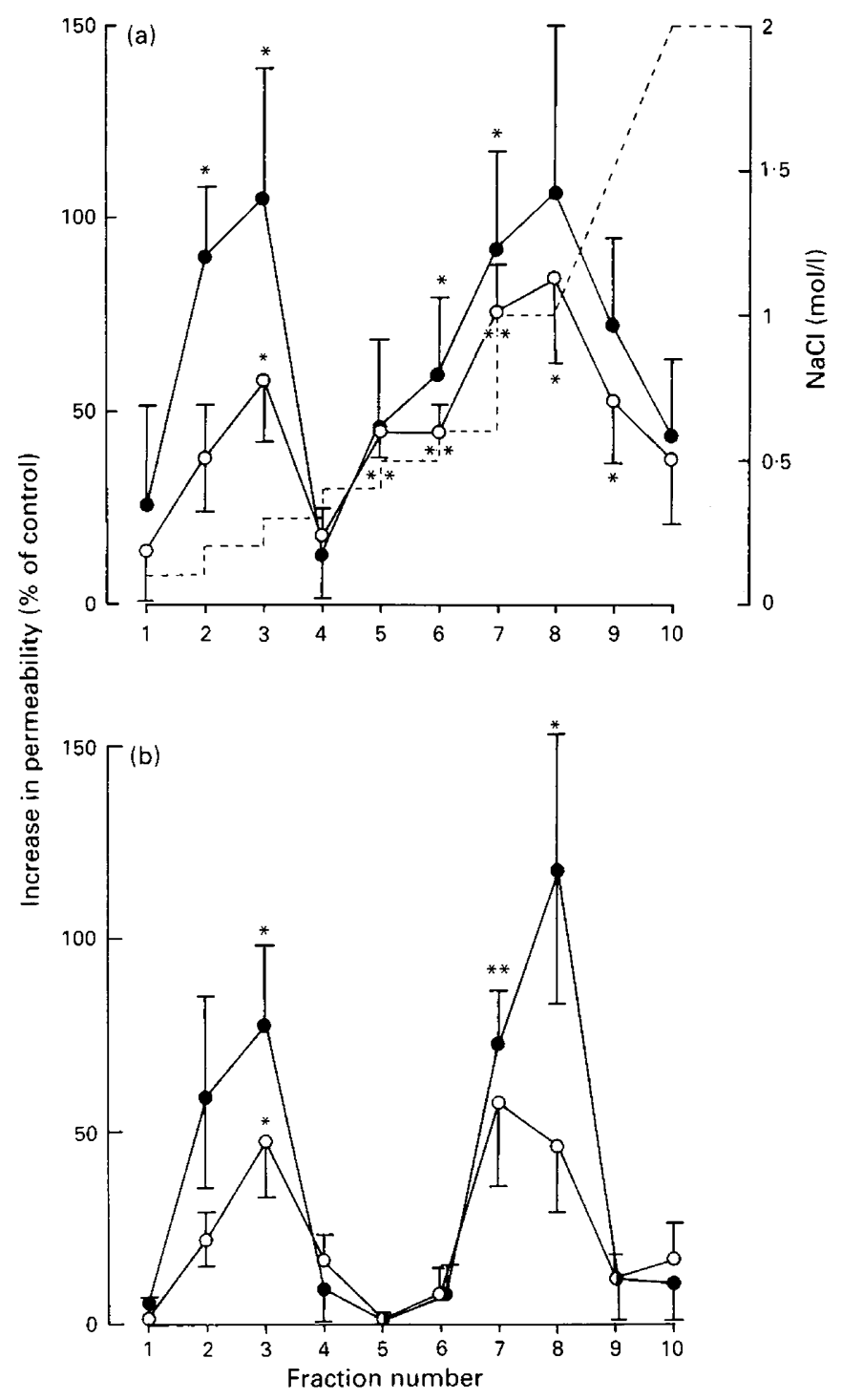

Fig. 1. Permeability-increasing activities of the high-performance liquid chromatography fractions of (a) rat testicular interstitial fluid (IF) and (b) medium conditioned by polymorphonuclear leucocytes; $(--)$ profile of the $\mathrm{NaCl}$ gradient. The effect of each fraction on the uptake of intravenously injected ${ }^{125}$ I-labelled human chorionic gonadotrophin (hCG) $(O)$ and on IF volume (-) was tested in the presence or absence (control) of $5 \mathrm{ng}$ of hCG. Each point represents the mean \pm s.e.m. of five chromatographies. Student's $t$ test was used for statistical comparisons; ${ }^{* *} P<0 \cdot 01,{ }^{*} P<0 \cdot 05$, in comparison with the corresponding control fractions.

gels were removed after incubations by centrifugation and the supernatants were tested for permeability activity as described above.

Histological examination. The volume densities of PMNs in the interstitial space and blood vessels in the testes were analysed in two groups of three rats. In the first group, the right testes were injected with $150 \mu$ l of the HPLCfraction 2 (see Fig. 3) containing 5 ng of the 'modified' hCG in PBS and the left testes received only PBS. In the second group, the right testes of the rats received $5 \mathrm{ng}$ of hCG in $150 \mu \mathrm{l}$ of the corresponding control HPLC fraction 2 (see Fig. 3) and the left testes received only PBS. All the animals were killed $4.5 \mathrm{~h}$ later, and the testes were removed, fixed in neutral buffered $4 \%$ formaldehyde, embedded in paraffin and sectioned at $1 \mu \mathrm{m}$. Two sections per testis were 


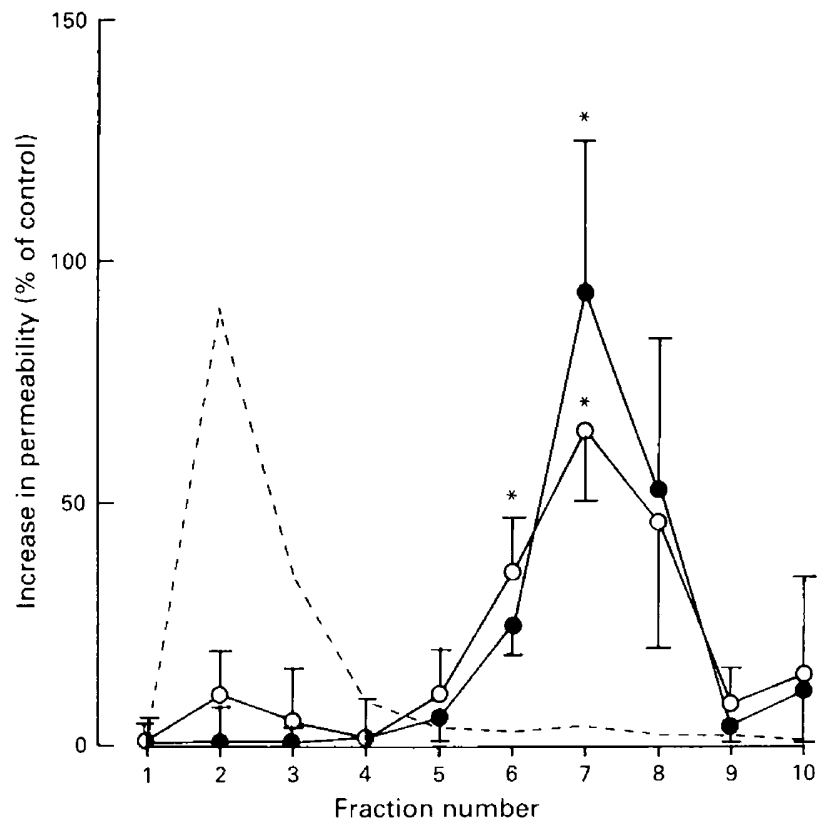

Fig. 2. Rechromatography of fraction 7-8 of medium conditioned by polymorphonuclear leucocytes on high-performance liquid chromatography (HPLC). Fractions 7-8 were pooled and rechromatographed on HPLC and each fraction was tested for the effect on the uptake of intravenously injected ${ }^{125}$ I-labelled human chorionic gonadotrophin (hCG) $(O)$ and volume of rat interstitial fluid (O) in the presence or absence (control) of $5 \mathrm{ng}$ of hCG. The results are presented as percentage of corresponding control fractions. Each point represents the mean \pm s.e.m. of five chromatographies; $(--)$ elution profile of hCG. Student's $t$ test was used for statistical comparisons; ${ }^{*} P<0.05$ in comparison with the corresponding control fractions.

stained with haematoxylin-eosin and the volume densities of PMNs in 50 fields per section were analysed by pointcounting method at magnification of $\times 700$ using a fine square lattice mounted in the eyepiece of a light microscope.

Statistical analyses. Student's $t$ and Mann-Whitney $U$ tests were used for statistical comparisons.

\section{Results}

\section{HPLC of IF and PMN-conditioned medium and the permeability-increasing activity of the fractions}

HPLC of IF revealed two broad peaks with maxima at fractions $2-3$ and 7-8, which with $5 \mathrm{ng}$ hCG were capable of causing a distinct increase in testicular vasopermeability (Fig. 1a). No increase in the permeability over the PBS-treated testes was seen if the testes were injected with the sample alone (control). Similarly, two peaks with maxima at the same fractions ( $2-3$ and $7-8)$ were seen when the PMN-conditioned medium was fractionated on HPLC (Fig. 1b). The latter activity peak was, however, sharper and separated from the former one with an interval of three nonactive fractions (4-6).

\section{Increase in permeability arising from conversion of hCG}

To elucidate whether hCG itself is converted so as to produce the increase in permeability, it was incubated in the HPLC-fractions 7-8 of the PMN-conditioned medium, purified subsequent to the incubation on HPLC and tested for permeability-increasing activity; hCG eluted from the HPLC-column as a sharp peak (Fig. 2) which, however, overlapped completely with the first 

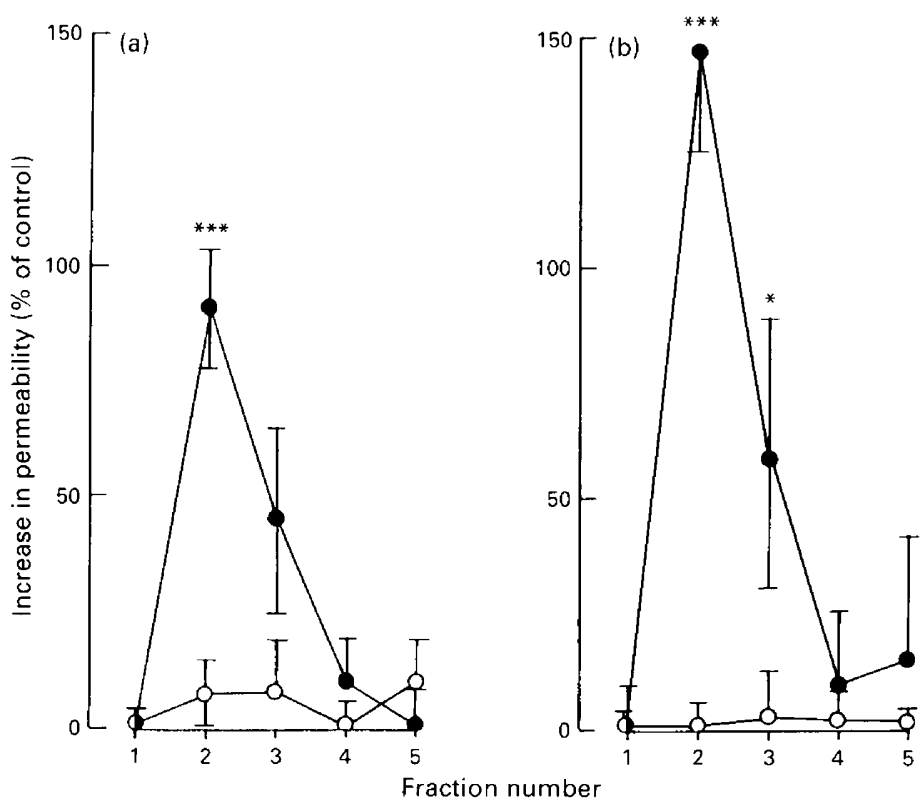

Fig. 3. Permeability-increasing activity of the 'modified' human chorionic gonadotrophin (hCG). The fractions containing 'modified' $\mathrm{hCG}(\boldsymbol{O})$ and the control fractions containing native hCG $(O)$ were injected intratesticularly and the effects on vasopermeability were tested by measuring (a) the uptake of intravenously injected ${ }^{125} \mathrm{I}-\mathrm{hCG}$ and (b) volume of rat interstitial fluid. The results are presented as percentages of PBS-injected control testes. Each point represents the mean \pm s.e.m. of 6-8 chromatographies. The Mann-Whitney $U$ test was used for statistical comparisons; ${ }^{* * *} P<0.001,{ }^{*} P<0.05$ in comparisons with the corresponding control fractions.

activity peak of the PMN-conditioned medium at fractions $2-3$, but was fully separated from the second one at fractions $7-8$. When fractions $7-8$ were pooled and rechromatographed, the activity appeared again at fractions 7-8 and was fully separated from hCG (Fig. 2). We took advantage of this result and incubated hCG with the HPLC fractions 7 and 8 of the PMN-conditioned medium for $2 \mathrm{~h}$ at $35^{\circ} \mathrm{C}$, subjected the incubation medium to HPLC and tested fractions 1-5 for the permeability-increasing activity without adding hCG to the fractions. The control incubation carried out in parallel without hCG was also subjected to HPLC, but the corresponding fractions were tested with hCG for permeability-increasing activity.

There was a sharp activity peak corresponding to the elution position of hCG, fractions 2-3 (Fig. 3). When native hCG was added to fractions $1-5$ of the control chromatography, no permeability-increasing activity was detected (Fig. 3), indicating that hCG itself is modified in the incubation to produce the increase in permeability.

To confirm that the 'modified' $\mathrm{hCG}$ is responsible for the permeability-increasing effect, hCG was incubated with the HPLC fractions 7-8 of the PMN-conditioned medium and the medium was subjected to HPLC. Subsequently, fraction 2 containing the modified hCG was treated with antihCG Sepharose 4B or control Sepharose and assayed for permeability-increasing activity. The samples treated with anti-hCG Sepharose 4B had lost virtually all their activity, while no change had occurred in controls (Fig. 4).

\section{Volume density analysis of PMNs in the testes after intratesticular treatment with modified hCG}

Intratesticular injection with $5 \mathrm{ng}$ of modified hCG in $150 \mu \mathrm{l}$ of PBS caused a significant accumulation of $\mathrm{PMNs}$ in postcapillary venules and in the interstitium compared with the controls, 

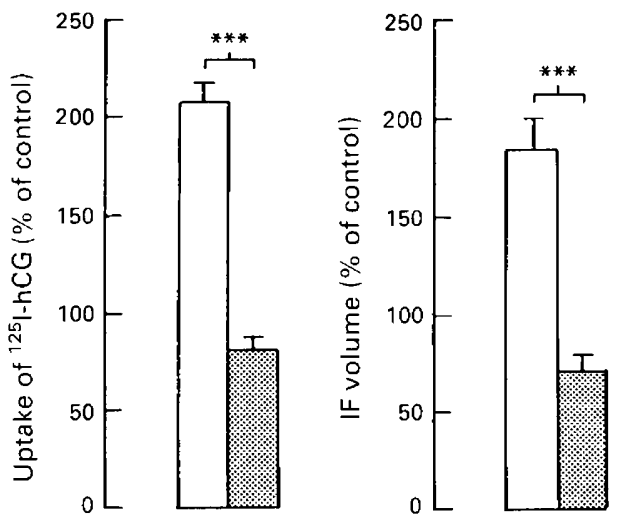

Fig. 4. Effect of the anti-hCG Sepharose $4 B$ treatment on the uptake in rat testes of intravenously injected ${ }^{125}$ I-labelled human chorionic gonadotrophin (hCG) and volume of interstitial fluid (IF) after intratesticular injection of the HPLC fraction containing the 'modified' hCG. The fraction containing $5 \mathrm{ng}$ of the 'modified' hCG/150 $\mu$ l of PBS was treated for $12 \mathrm{~h}$ with control Sepharose 4B ( $\square$ ) or anti-hCG Sepharose 4B (圈) and tested for the permeability response. Each column represents the mean \pm s.e.m. of 5-7 animals. The Mann-Whitney $U$ test was used for statistical comparisons; ${ }^{* * *} P<0 \cdot 001$.

in which the testes were injected with the same amount of native hCG in the corresponding control fraction or with PBS alone (Fig. 5).

\section{Discussion}

We have earlier suggested that hCG interacts with some component(s) present in testicular IF and PMN-conditioned medium to produce a factor that increases the testicular permeability via a PMN-mediated process, and that the component(s) interacting with hCG in IF possibly originates in neutrophils (Veijola \& Rajaniemi, 1986, 1989). The present results show that both IF and PMNconditioned medium probably contain two components of different charge, capable of interacting with hCG to produce the permeability response. The fact that the IF-components elute from the HPLC column with $\mathrm{NaCl}$ concentrations similar to those in the PMN-conditioned medium supports our earlier suggestion that they probably originate in neutrophils in the testes. The nature of these components was not explored in the present study. The components may, however, be proteins, as boiling IF (Veijola \& Rajaniemi, 1986) and PMN-conditioned medium for $10 \mathrm{~min}$ inactivates them. Whether they represent one protein with different isoelectric points remains to be clarified.

Our present results also suggest that it is likely that hCG itself is modified in the incubation to give rise to the permeability response. There are two lines of evidence to support this conclusion. First, hCG incubated with the HPLC fractions 7-8 of the PMN-conditioned medium and subsequently purified by HPLC was capable of causing the permeability response when given alone in PBS. The same amount of native hCG given in PBS or control fractions produced no permeability response within $4 \cdot 5 \mathrm{~h}$. Secondly, when the modified hCG was removed from the purified preparation with solid anti-hCG Sepharose 4B, the permeability response disappeared.

The modified hCG produced the permeability response via a PMN-mediated process, as a marked accumulation of PMNs occurred in the postcapillary venules and interstitium simultaneously with the increase in permeability. Thus, one explanation for the phenomenon is that hCG itself is converted to a chemotactically active form during the incubation. The hCG structure is, however, not drastically changed after exposure to the IF or PMN-conditioned medium 

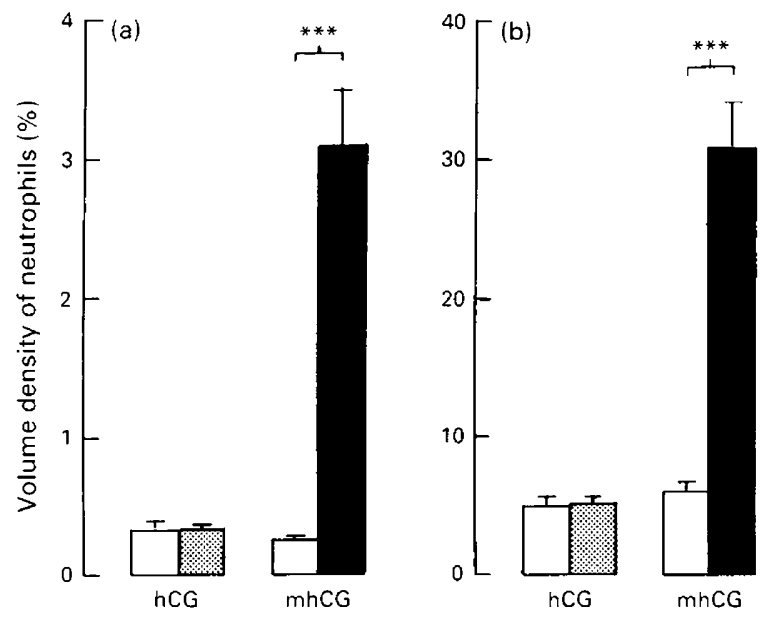

Fig. 5. Volume density of polymorphonuclear leucocytes (a) in rat testicular interstitium, expressed as a percentage of the interstitial space volume, and (b) in blood vessels, expressed as a percentage of blood vessel volume. The left testes $(\square)$ were injected with $150 \mu l$ of phosphatebuffered saline (PBS) and the right testes with the same volume of PBS-diluted HPLC fraction containing $5 \mathrm{ng}$ of the 'modified' human chorionic gonadotrophin ( $\mathbf{\square}, \mathrm{mhCG})$ or control

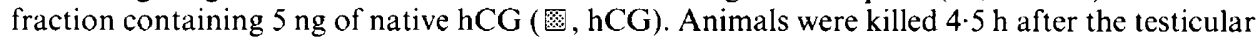
treatments. Each column represents the mean \pm s.e.m. of three animals; ${ }^{* * *} P<0 \cdot 001$.

components as it migrates with the same velocity on the one-dimensional SDS-PAGE and binds to $\mathrm{LH} / \mathrm{CG}$ receptor with the same affinity as native $\mathrm{hCG}$ (data to be published). Conformational changes resulting, for example, from partial reduction of disulphide bridges or subtle changes in the peptide core or carbohydrate structure cannot be ruled out. The lack of detectable changes in hCG structure does not suggest conversion of hCG to leucoattractant, but it does not fully exclude this possibility. The final conclusion of the possible leucoattractant nature of the 'modified' hCG can be made by directly assaying it for chemotactic activity using, for example, the Boyden chamber (Boyden, 1962). The alternative explanation is that the modified hCG gives rise to production of leucoattractant in the Leydig cells or some other testicular cell type, which secondarily elicits its permeability response via neutrophil extravasation. The Leydig cells are the most likely source of such a chemoattractant as they are the only known target cell for hCG in the testis and they are known to secrete chemoattractants (Cooke et al., 1984). Other cell types such as macrophages, which are known to produce leucoattractants (Hunninghake et al., 1978; Reynolds, 1983; Cunha \& Ferreira, 1986) may also be involved. However, the findings by Setchell \& Rommerts (1985) and Damber et al. (1987), showing that functionally intact Leydig cells are required to elicit the permeability response to subcutaneously injected hCG, indicate that Leydig cells are the source of the chemoattractants.

Our results provide the first evidence that hCG interacts in IF and PMN-conditioned medium with two components that give rise to the vasopermeability response. They suggest that hCG itself is modified in the interaction with these components so that it gains the permeability-increasing activity. The permeability response to the modified hCG is mediated by neutrophils, raising the possibility that the modified hCG is itself a leucoattractant or that it elicits production of leucoattractant.

We thank A. Kuha, P. Peronius, E. Hiltunen, S. Martti and E. Oja for expert technical and secretarial assistance. 


\section{References}

Bergh, A., Widmark, A., Damber, J.-E. \& Cajander, S. (1986) Are leucocytes involved in the human chorionic gonadotropin induced increase in testicular vascular permeability? Endocrinology 119, 586-590.

Boyden, S.V. (1962) The chemotactic effect of a mixture of antibody and antigen on polymorphonuclear leukocytes. J. exp. Med. 115, 453-466.

Cooke, B.A., Dix, C.J., Habberfield, A.D. \& Sullivan, M.H. (1984) Control of steroidogenesis in Leydig cells: roles of $\mathrm{Ca}^{2+}$ and lipoxygenase products in $\mathrm{LH}$ and LHRH agonist action. Ann. N.Y. Acad. Sci. 438, 269-282.

Cunha, F.Q. \& Ferreira, S.H. (1986) The release of a neutrophil chemotactic factor from peritoneal macrophages by endotoxin inhibition by glucocorticoids. Eur. J. Pharmacol. 129, 65-76.

Damber, J.-E., Bergh, A. \& Widmark, A. (1987) Testicular blood flow and microcirculation in rats after treatment with ethane dimethyl sulfonate (EDS). Biol. Reprod. 37, 1291-1296.

Damber, J.-E., Selstam, G. \& Wang, J. (1981) Inhibitory effect of estradiol-17\% on hCG-induced increment of testicular blood flow and plasma testosterone concentration in rats. Biol. Reprod. 25, 555-559.

Greenwood, F.E., Hunter, W.M. \& Glover, J.S. (1963) The preparation of ${ }^{131}$ I-labeled human growth hormone of high specific radioactivity. Biochem. J.89, 114-123.

Hunninghake, G.W., Gallin, J.I. \& Fauci, A.S. (1978) Immunologic reactivity of the lung. The in vivo and in vitro generation of a neutrophil chemotactic factor by alveolar macrophages. Am. Rev. Resp. Dis. 117, 15-23.

Ketelslegers, J.M., Knott, G.D. \& Catt, K.J. (1975) Kinetics of gonadotropin binding by receptors of the rat testis. Analysis by a nonlinear curve fitting method. Biochemistry 14, 3075-3085.

Loukusa, A.-K., Veijola, M. \& Rajaniemi, H. (1990) Plasminogen activator is involved in the hCGinduced neutrophil extravasation and vasopermeability increase in the rat testis. Int. J. Androl. 13, $306-314$

Markkanen, S., Töllikkö, K., Jääskeläinen, K. \& Rajaniemi, H. (1980) Effect of radioiodination on structural integrity, receptor and antibody binding activity and circulatory behavior of human choriongonadotropin. Hormone Res. 12, 32-45.

Metsikkö, K. \& Rajaniemi, H. (1980) Purification of luteinizing hormone receptor and its subunit structure. Biochem. biophys. Res. Commun. 95, 1730-1736.

Pande, J.K., Chowdhury, S.R., Dasgupta, P.R., Chowdhury, A.R. \& Kar, A.B. (1966) Biochemical composition of the rat testis fluid. Proc. Soc. Exp. Biol. Med. 121, 899-902.

Reynolds, H.Y. (1983) Lung inflammation: role of endogenous chemotactic factors in attracting polymorphonuclear granulocytes. Am. Rev. Resp. Dis. 127, S16-S25.

Setchell, B.P. \& Rommerts, F.F.G. (1985) The importance of Leydig cells in the vascular response to hCG in rats. Int. J. Androl. 8, 436 440.

Setchell, B.P. \& Sharpe, R.M. (1981) Effect of injected human chorionic gonadotropin on capillary permeability, extracellular fluid volume and the flow of lymph and blood in the testes of rats. J. Endocr. 91, 245-254.

Sharpe, R.M. (1979) Gonadotrophin-induced accumulation of interstitial fluid in the rat testis. J. Reprod. Fert. 55, 365-371.

Sharpe, R.M. (1981) The importance of testicular interstitial fluid accumulation in the transport of injected hCG to the Leydig cell. Int. J. Androl. 4, 64-74.

Veijola, M. \& Rajaniemi, H. (1986) Luteinizing hormones activate a factor(s) in testicular interstitial fluid which increases testicular vascular permeability. Mol. Cell. Endocr. 45, 113-118.

Veijola, M. \& Rajaniemi, H. (1989) Interaction of hCG with testicular interstitial fluid produces leucotactic factor(s). Int. J. Androl. 12, 307-317.

Veijola, M., Kellokumpu, S. \& Rajaniemi, H. (1984) The effect of varying doses of hCG on the in vivo uptake by rat testis and serum testosterone response. Hormone Res. 19, 191-199.

Widmark, A., Damber, J.-E. \& Bergh, A. (1986) Relationship between human chorionic gonadotrophininduced changes in testicular microcirculation and the formation of testicular interstitial fluid. J. Endocr. $109,419-425$.

Received 19 November 1990 